\title{
Prediction of Human Drug-Drug Interactions
}

\author{
Created by: Qulrika Simonsson
}

Version received: 14 April 2020

check for

updates

\begin{abstract}
Patients suffering from complex diseases (infectious diseases, oncology) or several conditions (comorbidities) require combination therapies of different drugs. Combining different drugs will potentially lead to drug-drug interactions, influencing the efficacy and safety of the treatment. Development of novel drugs that are intended to be used in combination should consider drug-drug interactions as early as possible. Even with limited data, drug-drug interactions in humans can be predicted using model-informed drug discovery and development (MID3). The concept to utilize and integrate data from in vitro combination experiments combined with preclinical in vivo data on the exposure-response relationships of the drugs in combination through a computational modelinformed approach, is introduced here with tuberculosis as case study.
\end{abstract}

Some diseases require combination therapy for efficacious and safe therapy. For example tuberculosis requires treatment with at least three different antibiotics to prevent resistance development of the pathogen against one or more of the antibiotics. Combination therapy inherently has the risk of interactions between the individual drugs, so called drug-drug interactions or DDIs. The same holds for patients with different diseases (comorbidities) that require treatment with different drugs at the same time.

Drug-drug interactions between drugs that are intended to be used in combination should be considered as early as possible in drug development [ $\underline{1}$. The prediction of DDIs from preclinical data will improve the ability to predict the total efficacy of the combination in relation to the drugs in monotherapy, as well as compared to expected additivity, i.e., the sum of all effects from the drugs when given alone. Drug-drug interactions that result in less efficacy in the combination than in a combination with one less drug should be avoided. However, combinations that result in an efficacy less than the expected additivity, but still result in more efficacy than when one drug is omitted, can be considered. Drug-drug interactions can relate to both pharmacokinetic (PK) interactions, i.e., one drug (the perpetrator) impacting the absorption, distribution, metabolism, or excretion of another drug (the victim), or pharmacodynamic (PD) interactions, i.e., the perpetrator impacting the potency or efficacy of the victim drug.

Regulatory guidelines on the investigation of DDIs are brief about the use of in vitro data. An innovative approach in the field, model-informed drug discovery and development (MID3) is a promising way of integrating prior information [2] [3] [4] . Knowledge on the relevant mechanisms of, e.g., metabolism combined with in vitro data can be leveraged to decide on suitable combinations of drugs without extensive experimentation [ㄷ][]. B. Both in vitro studies as well as animal experiments can be utilized to assess the potential for PK DDIs []. In vitro studies make use of metabolically active hepatocytes or cells overexpressing drug transporters to determine the PK interaction potential of a new drug []. When studying DDIs in preclinical

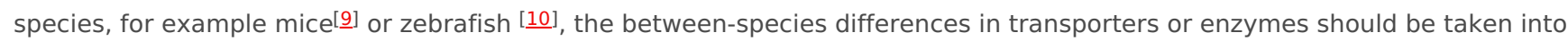
account [11].

Pharmacokinetic DDIs mostly impact drug clearance by the induction or inhibition of metabolic enzymes like those from the cytochrome P450 (CYP) family and, to some extent, ATP-binding cassette (ABC) transporters and transport proteins. Such an interaction by the perpetrator drug will greatly enhance or reduce the exposure of the victim drug. An example from tuberculosis therapy is the fact that rifampicin induces bedaquiline clearance 5 -fold, and should therefore not be combined for therapy[12]. Because bedaquiline has a very long terminal half-life, potential DDIs are difficult to identify using traditional methods, whereas properly designed experiments and quantitative modeling are necessary to elucidate such interactions $[\underline{13}]$.

Drug distribution can also be impacted because of the induction or inhibition of drug transporters like the permeability glycoprotein (P-gp), which is present on the canalicular membrane and blood-brain barrier, among others. Physiology based pharmacokinetic modeling (PBPK) can be very successful to predict metabolic DDIs, and specific DDI studies can be assisted by modeling and simulations [14].

Some anti-tuberculosis drugs are reported to be substrates for different hepatic enzymes or known to be inducers or inhibitors of

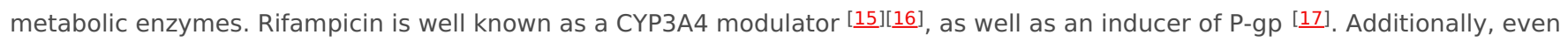
though the effect of clofazimine on CYP3A4 and P-gp is still unclear, clofazimine has been shown to delay the time taken to reach $\mathrm{C}_{\max }$ of rifampicin [18]. Horita et al. studied the effects of anti-TB and antiretroviral drugs on CYP3A4 and P-gp, and they found that clofazimine exhibits weak inductive effects on CYP3A4 [밀. Furthermore, the co-administration of bedaquiline and clofazimine has been reported to increase the risk of QT prolongation potentially resulting in cardiac adverse events [20][21]. As described above, these potential DDIs can be predicted from in vitro data through, for example, in vitro-in vivo scaling ${ }^{[22]}$ or 
PBPK [23]. A transcription/translation model and a PBPK model have been developed to predict rifampicin-induced DDIs with reasonable accuracy [24].

In contrast to PK interactions, due to clearly defined processes of absorption, distribution, metabolism, and excretion, PD interactions are harder to investigate and quantify. This is because, since a clinical DDI study has to study the drugs both alone and in combination, the number of arms in the study will substantially increase when studying three or more interacting drugs. The Greco model [드] , which is derived from Loewe additivity, was developed to assess PD interactions. However, such a model suffers from being limited to interactions between only two drugs. On the other hand, the general pharmacodynamic interaction (GPDI) model overcomes this limitation, in addition to being flexible to different drug interaction data without requiring knowledge on the modes of action of the studied drugs [ㄷ]. The GPDI model-based approach proposes a PD interaction to be quantifiable, as multidirectional shifts in drug efficacy $\left(E_{\max }\right)$ or potency $\left(E_{50}\right)$ and explicates the drugs' role as victim, perpetrator or even both at the same time. The GPDI model has been utilized along with the multistate tuberculosis pharmacometric (MTP) model [그] to develop a model-informed preclinical approach for the prediction of PD interactions [28]. The MTP-GPDI model has been further employed to successfully evaluate and quantify the PD interactions of anti-TB drug combinations in mice [29]. Furthermore, it has been demonstrated that the GPDI model outperforms conventional methods in the evaluation of PD interactions for TB drugs [으.

It is clear that the need for a combination therapy of TB could potentially result in DDIs in the clinic. It is therefore essential to quantitatively understand the DDIs, both PK- and PD-interactions, as early as possible in drug development. Utilizing data from in vitro combination experiments combined with preclinical in vivo data on the exposure-response relationships of the drugs in combination and early clinical data, will inform on which combinations of drugs at which doses are efficacious and safe for patients. This quantitative integration of data and translation to the clinic is possible through the MID3 model-informed framework.

\section{References}

1. Rob C. Van Wijk; Rami Ayoun Alsoud; Hans Lennernäs; Ulrika Sh Simonsson; Model-Informed Drug Discovery and Development Strategy for the Rapid Development of Anti-Tuberculosis Drug Combinations. Applied Sciences 2020, 10, 2376, 10.3390/app10072376.

2. Efpia Mid3 Workgroup; Scott Marshall; R Burghaus; V Cosson; Sya Cheung; M Chenel; O Dellapasqua; N Frey; B Hamrén; L Harnisch; et al.F IvanowT KerbuschJ LippertPa MilliganS RohouA StaabJI SteimerC TornøeSag Visser Good Practices in Model-Informed Drug Discovery and Development: Practice, Application, and Documentation. CPT: Pharmacometrics \& Systems Pharmacology 2016, 5, 93-122, 10.1002/psp4.12049.

3. Scott Marshall; Rajanikanth Madabushi; Efthymios Manolis; Kevin Krudys; Alexander Staab; Kevin Dykstra; Sandra A.G. Visser; Model-Informed Drug Discovery and Development: Current Industry Good Practice and Regulatory Expectations and Future Perspectives. CPT: Pharmacometrics \& Systems Pharmacology 2019, 8, 87-96, 10.1002/psp4.12372.

4. Efthymios Manolis; Jacob Brogren; Susan Cole; Justin L. Hay; Anna Nordmark; Kristin E. Karlsson; Frederike Lentz; Norbert Benda; Gaby Wangorsch; Gerard Pons; et al.Wei ZhaoValeria GiganteFrancesca Seronejoseph F. StandingAristides DokoumetzidisJuha VakkilainenMichiel Van Den HeuvelV. Mangas-Sanjuanjohannes TaminiauEssam KerwashDavid KhanFlora Tshinanu Musuambalne Skottheim Rusten Commentary on the MID3 Good Practices Paper. CPT: Pharmacometrics \& Systems Pharmacology 2017, 6, 416-417, 10.1002/psp4.12223.

5. European Medicine Agency; Guideline on the Investigation of Drug Interactions.European Medicine Agency 2012 , CPMP/EWP/560/95, na, https://www.ema.europa.eu/en/investigation-drug-interactions.

6. Pascal Schulthess; Rob C. Van Wijk; Elke H. J. Krekels; James W T Yates; Herman Spaink; Piet H. Van Der Graaf; Outside-In Systems Pharmacology Combines Innovative Computational Methods With High-Throughput Whole Vertebrate Studies. CPT: Pharmacometrics \& Systems Pharmacology 2018, 7, 285-287, 10.1002/psp4.12297.

7. J Kuhlmann; W Mück; Clinical-pharmacological strategies to assess drug interaction potential during drug development.. Drug Safety 2001, 24, 715-725.

8. Hugh A Barton; Yurong Lai; Theunis C Goosen; Hannah M. Jones; Ayman F El-Kattan; James R Gosset; Jian Lin; Manthena V. S. Varma; Model-based approaches to predict drug-drug interactions associated with hepatic uptake transporters: preclinical, clinical and beyond. Expert Opinion on Drug Metabolism \& Toxicology 2013, 9, 459-472, 10.1517/17425255.2013.759210.

9. Umesh Datta Gupta; Amit Kumar Singh; Animal models of tuberculosis: Lesson learnt.Indian Journal of Medical Research 2018, 147, 456-463, 10.4103/ijmr.ijmr_554_18.

10. Rob C Van Wijk; Astrid M Van; Elke Hj Krekels; Theo Verboom; Herman P Spaink; Ulrika Sh Simonsson; Piet H. Van; Der Sar; Der Graaf; Quantification of natural growth of two strains of Mycobacterium marinum for translational antituberculosis drug development.. Clinical and Translational Science 2020, Epub April, -, 10.1111/cts.12793.

11. X Chu; Kelly Bleasby; Raymond Evers; Species differences in drug transporters and implications for translating preclinical findings to humans. Expert Opinion on Drug Metabolism \& Toxicology2012, 9, 237-252, 10.1517/17425255.2013.741589.

12. Helen Mcllleron; Maxwell Tawanda Chirehwa; Current research toward optimizing dosing of first-line antituberculosis 
treatment. Expert Review of Anti-infective Therapy 2018, 17, 27-38, 10.1080/14787210.2019.1555031.

13. Elin M. Svensson; Chayan Acharya; Björn Clauson; Kelly E. Dooley; Mats O. Karlsson; Pharmacokinetic Interactions for Drugs with a Long Half-Life-Evidence for the Need of Model-Based Analysis.. The AAPS Journal 2016, 18, 171-9, 10.1208/s12248-015-9829-2.

14. Alison Margolskee; Adam Darwich; Xavier Pepin; Shriram M. Pathak; Michael B. Bolger; Leon Aarons; Amin RostamiHodjegan; Jonas Angstenberger; Franziska Graf; Loic Laplanche; et al.Thomas MüllerSara CarlertPankaj DagaDónal MurphyChrister TannergrenMohammed YasinSusanne Greschat-SchadeWolfgang MückU. MuensterDorina Van Der MeyKerstin Julia FrankRichard LloydLieve AdriaenssenJan BevernageLoeckie De ZwartDominique SwertsChristophe TistaertAn Van Den BerghAchiel Van PeerStefania BeatoAnh-Thu Nguyen-TrungJoanne BennettMark McAllisterMei WongPatricia ZaneCéline OllierPascale VicatMarkus KolhmannAlexander MarkerPriscilla BrunFlorent MazuirStéphane BeillesMarta VenczelXavier BoulencPetra LoosHans LennernäsBertil Abrahamsson IMI - oral biopharmaceutics tools project - evaluation of bottom-up PBPK prediction success part 1: Characterisation of the OrBiTo database of compounds. European Journal of Pharmaceutical Sciences 2017, 96, 598-609, 10.1016/j.ejps.2016.09.027.

15. Jiezhong Chen; Kenneth Raymond; Roles of rifampicin in drug-drug interactions: underlying molecular mechanisms involving the nuclear pregnane X receptor. Annals of Clinical Microbiology and Antimicrobials 2006, 5, 3-3, 10.1186/1476-0711-5-3.

16. Niladri Chattopadhyay; Tobias Kanacher; Manuela Casjens; Sebastian Frechen; Sandra Ligges; Torsten Zimmermann; Antje Rottmann; Bart Ploeger; Joachim Höchel; Marcus-Hillert Schultze-Mosgau; et al. CYP3A4-mediated effects of rifampicin on the pharmacokinetics of vilaprisan and its UGT1A1-mediated effects on bilirubin glucuronidation in humans. British Journal of Clinical Pharmacology 2018, 84, 2857-2866, 10.1111/bcp.13750.

17. Bernd Greiner; Michel Eichelbaum; Péter Fritz; Hans-Peter Kreichgauer; Oliver Von Richter; Johannes Zundler; Heyo K. Kroemer; The role of intestinal P-glycoprotein in the interaction of digoxin and rifampin. Journal of Clinical Investigation 1999, 104, 147-153, 10.1172/jci6663.

18. J. M. Mehta; I. S. Gandhi; S. B. Sane; M. N. Wamburkar; Effect of clofazimine and dapsone on rifampicin (Lositril) pharmacokinetics in multibacillary and paucibacillary leprosy cases. Leprosy Review 1986, 57, 67-76, 10.5935/03057518.19860097.

19. Yasuhiro Horita; Norio Doi; Comparative Study of the Effects of Antituberculosis Drugs and Antiretroviral Drugs on Cytochrome P450 3A4 and P-Glycoprotein. Antimicrobial Agents and Chemotherapy 2014, 58, 3168-3176, 10.1128/AAC.02278-13.

20. Dannemann, B.; Bakare, N.; De Marez, T.; Lounis, N.; Van Heeswijk, R.P.G.; Meyvisch, P.; Haxaire-Theeuwes, M.; Andries, K.; Everitt, D.; Upton, A; et al. Corrected QT Interval (QTCF) Prolongation in a Phase 2 Open-Label Trial of TMC207 plus Background Regimen as Treatment for MDR-TB: Effect of Co-Administration with Clofazimine. Proceedings of the Abstract at 52nd Interscience Conference on Antimicrobial Agents and Chemotherapy, San Francisco, CA, USA, 9-12 September 2012 2012, 52, -.

21. Alexander S Pym; Andreas H. Diacon; Shen-Jie Tang; Francesca Conradie; Manfred Danilovits; Charoen Chuchottaworn; Irina Vasilyeva; Koen Andries; Nyasha Bakare; Tine De Marez; et al.Myriam Haxaire-TheeuwesNacer LounisPaul MeyvischBen Van BaelenRolf P.G. Van HeeswijkBrian Dannemann Bedaquiline in the treatment of multidrug- and extensively drug-resistant tuberculosis. European Respiratory Journal 2015, 47, 564-574, 10.1183/13993003.00724-2015.

22. T Iwatsubo; H Suzuki; N Shimada; K Chiba; T Ishizaki; C E Green; C A Tyson; T Yokoi; T Kamataki; Y Sugiyama; et al. Prediction of in vivo hepatic metabolic clearance of YM796 from in vitro data by use of human liver microsomes and recombinant P-450 isozymes.. Journal of Pharmacology and Experimental Therapeutics 1997, 282, 909-919.

23. P Zhao; L Zhang; J A Grillo; Q Liu; J M Bullock; Y J Moon; P Song; S S Brar; R Madabushi; T C Wu; et al.B P BoothN A RahmanK S ReynoldsE Gil BerglundL J LeskoShiew-Mei Huang Applications of Physiologically Based Pharmacokinetic (PBPK) Modeling and Simulation During Regulatory Review. Clinical Pharmacology \& Therapeutics 2010, 89, 259-267, 10.1038/clpt.2010.298.

24. Fumiyoshi Yamashita; Yukako Sasa; Shuya Yoshida; Akihiro Hisaka; Yoshiyuki Asai; Hiroaki Kitano; Mitsuru Hashida; Hiroshi Suzuki; Modeling of Rifampicin-Induced CYP3A4 Activation Dynamics for the Prediction of Clinical Drug-Drug Interactions from In Vitro Data. PLOS ONE 2013, 8, e70330, 10.1371/journal.pone.0070330.

25. W R Greco; H S Park; Y M Rustum; Application of a new approach for the quantitation of drug synergism to the combination of cis-diamminedichloroplatinum and 1-beta-D-arabinofuranosylcytosine.. Cancer Research 1990, 50 , 5318-5327.

26. Sebastian Georg Wicha; Chunli Chen; Oskar Clewe; Ulrika Simonsson; A general pharmacodynamic interaction model identifies perpetrators and victims in drug interactions. Nature Communications 2017, 8, 2129, 10.1038/s41467017-01929-y.

27. Oskar Clewe; Linda Aulin; Yanmin Hu; Anthony R. M. Coates; Ulrika Simonsson; A multistate tuberculosis pharmacometric model: a framework for studying anti-tubercular drug effects in vitro.. Journal of Antimicrobial Chemotherapy 2015, 71, 964-74, 10.1093/jac/dkv416.

28. Oskar Clewe; Sebastian G Wicha; Corné P De Vogel; Jurriaan E M De Steenwinkel; Ulrika Simonsson; A model- 
informed preclinical approach for prediction of clinical pharmacodynamic interactions of anti-TB drug combinations. Journal of Antimicrobial Chemotherapy 2017, 73, 437-447, 10.1093/jac/dkx380.

29. Chunli Chen; Sebastian G. Wicha; Gerjo J. De Knegt; Fatima Ortega; Laura Alameda; Veronica Sousa; Jurriaan E. M. De Steenwinkel; Ulrika Simonsson; Assessing Pharmacodynamic Interactions in Mice Using the Multistate Tuberculosis Pharmacometric and General Pharmacodynamic Interaction Models. CPT: Pharmacometrics \& Systems Pharmacology 2017, 6, 787-797, 10.1002/psp4.12226.

30. Chunli Chen; Sebastian G. Wicha; Rikard Nordgren; Ulrika Simonsson; Comparisons of Analysis Methods for Assessment of Pharmacodynamic Interactions Including Design Recommendations. The AAPS Journal 2018, 20, 77, 10.1208/s12248-018-0239-0.

\section{Keywords}

drug-drug interaction; drug development; pharmacokinetics; pharmacodynamics; translational pharmacology; tuberculosis; pharmacometrics; model-informed drug discovery and development; preclinical; clinical 